\title{
Adhesive Small Bowel Obstruction, Case Report and Review of Literature
}

M. Maliki-Alaoui*, YZA. Khedid, T. Lachguer, I. ENNouhi, I. Maaouni, M. Gridda, N. Bahrou, M. Echarrab, M. ElAbsi, M. El Ounani, FH. El Alami, M. Amraoui, A. Errougani

Department of the Emergency Surgery (UCV) - Ibn Sina University Hospital, Rabat, Medical School, Mohammed V University in Rabat, Morocco

DOI: $10.36347 /$ sjmcr.2020.v08i09.006

| Received: 31.08.2020 | Accepted: 08.09.2020 | Published: 09.09.2020

*Corresponding author: Mohamed Maliki-Alaoui

\section{Abstract}

Taking into account the frequency, morbidity and mortality of peritoneal adhesion, they constitute a real public health problem in both developed and emerging countries. Small bowel obstruction (SBO) due to postoperative adhesions is their main presentation. We report a case of patient admitted to the emergency department after a 5 hours-onset small bowel obstruction with a history of appendectomy 5 years ago. According to the onset of symptoms, hemodynamic stability and normal blood tests, we choose a non-surgical management. With the hemodynamic deterioration non resolution of the obstruction the patient was operated. Postoperative adhesions are currently the main cause of acute small bowel obstruction. The most effective prevention way is the respect of the general principles of abdominal surgery.

Keywords: Adhesion, bowel, bridles, obstruction, postoperative, prevention.

Copyright @ 2020: This is an open-access article distributed under the terms of the Creative Commons Attribution license which permits unrestricted use, distribution, and reproduction in any medium for non-commercial use (NonCommercial, or CC-BY-NC) provided the original author and source are credited.

\section{INTRODUCTION}

Any aggression of the peritoneum; infection, irritation or surgery, causes an inflammatory reaction resulting in the formation of adhesions.

Almost a $1 / 3$ of patients who undergo a laparotomy are readmitted on average twice for adhesive complications. This include: acute bowel obstruction, chronic abdomino-pelvic pain and infertility [1]. Adhesions are also associated with operating difficulties during a subsequent surgery, with the risk of visceral lesions.

This therefore exposes health care system to considerable morbidity, mortality and economic burden. As the cost of health care continues to climb and the number of patients requiring surgical management increases as the population ages, the financial burden of adesions will continue to increase.

Over the past two decades, much research has been carried out to understand the biochemical and cellular phenomena of adhesions formation, their frequency, predisposing factors and prevention methods.

\section{Case Report}

A 40-year-old patient admitted to the emergency department for the management of diffuse abdominal pain, bloating, nausea and vomiting. She had a history of appendectomy 5 years ago. An abdominal X-ray (Fig-1) showed a typical pattern of small bowel obstruction. A dilated loops aspect in computed tomography led to the diagnosis of SBO. There were no signs of ischemia or necrosis. We first choose a nonsurgical management, according to the onset of symptoms (5 hours), hemodynamic stability and normal blood tests. That includes intra-venous hydration, painkillers, nasogastric tube and an X-ray control. With the hemodynamic deterioration, lactic acidosis and the clinical and radiological non resolution of the occlusion the patient was operated. Striction of the small bowel around an adhesion led to bowel distension (Fig-2-3). We performed a complete bowel adhesiolysis without resection and a bowel retrograde evacuation and placement. The patient was discharged from hospital on the 4th post-op day in excellent condition. 


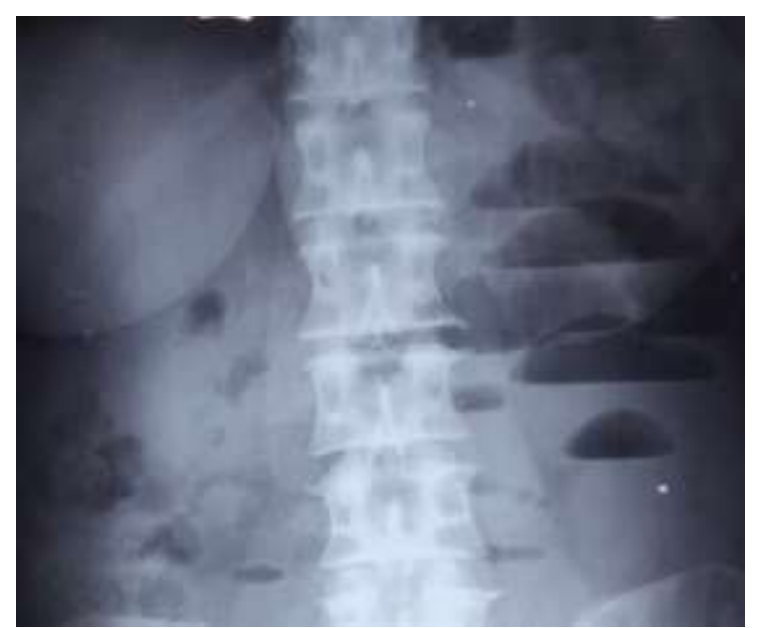

Fig-1: abdominal X-ray showing a typical pattern of small bowel obstruction

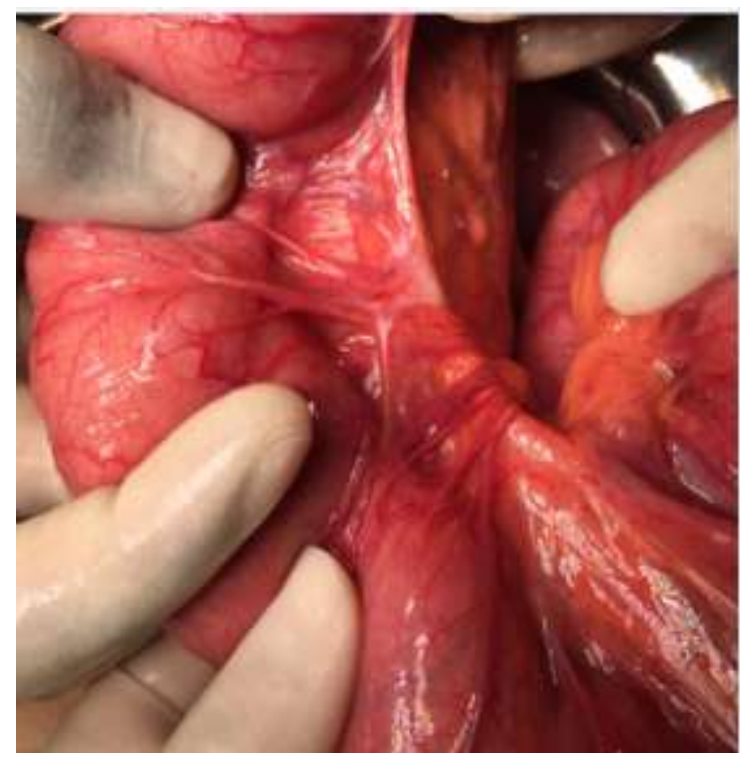

Fig-2: Surgical findings: peritoneal adhesion

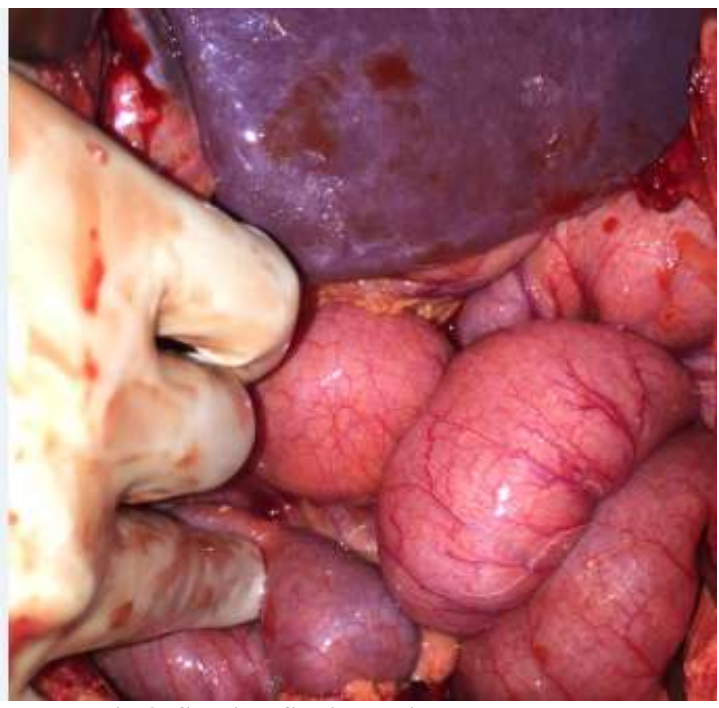

Fig-3: Surgical findings: dilated bowel loops

\section{DISCUSSION}

\section{A. Definition and importance of the problem}

Peritoneal adhesions correspond to fibrous formations developing between two digestive structures (two loops) or between a loop and the wall, or between the greater omentum and a surgically deperitonized area (operative scar, parietal peritoneum, uterine fundus). Thy can be a thin film of connective tissue, a thick fibrous bridge containing blood vessels, or a direct adhesion between two organ surfaces. Unlike adhesions, which are wider and more diffuse, bridles are fibrous, firm cylinders of varying length stretched across the abdominal cavity [2].

They have $80 \%$ surgical origin. If not, they are of congenital or inflammatory origin. They form as early as 72 hours after surgery and reach a maximum in intensity between the 10th and 15th postoperative day [3]. In the postoperative period, the areas where adhesions are found are often the incision, the greater omentum, the operative site and the small intestine. The incision and the surgical site are areas of major trauma.

Their formation and reformation can be a cause of significant morbidity, with multiple potential complications, many of which sometimes appear several years after the first laparotomy. It is considered that after any type of abdominal surgery, the percentage of patients with adhesions is between 93 and 100\% [4].

\section{B. Predisposing factors}

The most important one is the type of surgery and the extent of peritoneal damage: Colon and rectal surgeries and gynecologic surgeries are associated with a higher risk of complications from adhesions. Surgical techniques causing tissue trauma (electrocoagulation, resection of tissue by ultrasound, laser energy) [4].

Postoperative complications, peritonitis, emergency surgery. The existence of preliminary adhesions. Multiple previous laparotomies (> 3).

Untreated intra-abdominal inflammatory diseases. Penetrating abdominal trauma, especially gunshot wounds. Africans develop keloids more easily, so they may be more at risk of forming adhesions. Age less than 60 years.

Intra-peritoneal foreign bodies: any abdominal intervention is accompanied by "peritoneal pollution". [6]Radiation therapy and chemotherapy: bridles are often mentioned as secondary to pelvic irradiation. For intra - peritoneal chemotherapy, the data are contradictory since, experimentally, some products show efficacy in the prevention of bridles while clinically the importance of adhesions after such treatment has been highlighted [7]. 
$\mathrm{CO}_{2}$ pneumoperitoneum used in laparoscopy. Hemoperitoneum seems to potentiate the action of other factors.

\section{Clinical complications}

\section{Acute bowel obstruction}

Postoperative adhesions are currently the leading cause of acute small bowel obstruction with a relative prevalence of $32 \%$ [3]. Particularly dreadful complication:

For the patient: it is an unforeseeable emergency inevitably leading to hospitalization with the risk of emergency surgery in the following hours or days.

For the surgeon: lack of predictive factor before necrosis allowing to set the indication for surgery, whether clinical, biological or CT apart from a few signs of gravity. This surgery has elements of gravity due to the septic potential and the technical difficulties which increase the duration of the intervention and the risk of enterotomy. The intervention is not curative as shown by the rate of rehospitalizations and reoperations.

For the society, this pathology has a considerable social and financial impact, both for the surgical management itself and for the costs of hospitalization, convalescence and temporary professional disinsertion that this causes.

\section{Other clinical complications}

Accidental enterotomy is statistically the first recognized complication of adhesiolysis, with an incidence of approximately $20 \%$ on surgical revision, leading to significant postoperative mortality and morbidity (fistula, intestinal obstruction, abscess, respiratory complications). The number of previous laparotomies and obesity (BMI> $25 \mathrm{~kg} \mathrm{/} \mathrm{m2)} \mathrm{are}$ considered to be risk factors for intestinal damage.

Adhesions were incriminated in $25 \%$ of the patients who consulted for chronic pelvic pain and constitute a frequent reason $(10 \%)$ of consultation in gynecology [8]. These pains could be explained by the limitation of the movement of the viscera abnormally joined together. Some patients may be relieved after adhesiolysis. However, the causal relationship between peritoneal adhesions and chronic pain is not always clear.

Female sterility and ectopic pregnancy: by restricting the sweep of the tube fringes on the ovary. Peri-adnexal adhesions have been found in $20-30 \%$ of infertile women and the pregnancy rate significantly increased after adhesiolysis [9].
In our era of advanced laparoscopic surgery, adhesions have become even more important, frequently making this approach more difficult, if not impossible. Extensive adhesiolysis is often required, resulting in prolonged operating time, increased blood loss and other complications.

For patients with chronic kidney failure, adhesions can make peritoneal dialysis impossible.

\section{Prevention}

A study has shown that the use of a preventive method that is $25 \%$ efficient has saved 40 million€ in 10 years [1]. Several agents (mechanical barriers, chemical agents) have been tested in both animals and humans. In lak of an evidence based protocol of the use of these agents, the most effective way is the respect of the general principles of abdominal surgery (Halsted principles).

Any surgical technique that can reduce traumatic lesions of the peritoneum is recommended. Atraumatic manipulation of tissues, achieving careful hemostasis, parsimonious coagulation of the peritoneum, protection of the small bowel by moist fields, sparing use of sutures [10]. Frequent irrigation of the dissection area to avoid the formation of fibrinous deposits, excisions of peritoneal lesions with scissors and minimal use of monopolar electrocoagulation to avoid diffused thermal injuries, maximum resection of devitalized tissues , elimination of foreign bodies (talc, threads), fibrinous residues and blood clots before peritoneal closure [3].

The following are also recommended: the careful peritoneal cleansing of any peritonitis or hemoperitoneum using warmed physiological serum, remove any false membranes (without harsh), carry out the spotting of serous or mesenteric wounds, reposition the omentum over the intestinal loops and under the incision, avoid unnecessary draining or prefer inert materials (silicone).

Laparoscopy: its benefit on adhesions is directly correlated with the reduction in operating time and the pressure of the pneumoperitoneum, the humidification and heating of the insufflated $\mathrm{CO}_{2}$, the regular use of suction and washing, the parsimonious coagulation use and gentle manipulation of the intestinal loops and peritoneum [11]. Its theoretical advantages include: minimal incision of the parietal peritoneum, maximal reduction of microscopic foreign bodies, decreased bleeding and more precise dissection (especially for pelvic and rectal surgery) and the absence or minimization of handling beyond the operational zone. 
The Noble or Childs-Philips intestinal and mesenteric plication or fixation procedures are abandoned. Their effectiveness has not been established, they are fraught with serious complications, and subsequent operation for these patients is often extremely difficult.

\section{CONCLUSiON}

Adhesions are often responsible of direct complications (acute bowel obstruction, chronic abdominal pain, infertility) and indirect complications encountered during reoperation (difficult dissection, prolonged operating time). Prevention seems to be the first way to reduce this morbidity.

\section{REFERENCES}

1. Arung W, Meurisse M, Detry O. Pathophysiology and prevention of postoperative peritoneal adhesions. World J Gastroenterol. 2011Nov 7;17(41):4545-53.

2. Arung W. Intraperitoneal adhesions after open or laparoscopic abdominal procedure: experimental study in the rat. J Laparoendosc Adv Surg Tech A. 2012Sep 22;(7):651-7.

3. Maliki-Alaoui M. Les occlusions intestinales sur brides postopératoires, étude rétrospective à propos de 134 cas. 2014. Medical thesis.

4. Ouaïssi M. Post-operative adhesions after digestive surgery: Their incidence and prevention: Review of the literature. Journal of Visceral Surgery. 2012 Apr2;149(2):e104-e114.

5. Di Saverio. Bologna guidelines for management of ASBO: update of the evidence-based guidelines from the world society of emergency surgery. World Journal of Emergency Surgery. 2013;8:42.

6. Bakkum EA. Quantitative analysis of the inflammatory reaction surrounding sutures used in operative procedure and the relation to postsurgical adhesion formation. Biomaterials. 1995;16:1283- 1289.

7. Cubukcu A, Alponat A et Gonullu NN. An experimental study evaluating the effect of Mitomycin $\mathrm{C}$ on the prévention of postopérative intra-abdominal adhesions. $\mathrm{J}$ surg Res. 2001;96:163-166.

8. Sibert L, Safsaf A, Rigaud J. Approches symptomatiques des douleurs sexuelles chroniques. Prog Urol. 2010;20:967-72.

9. Marana R, Muzii L. Infertility and adhesions. In: Di Zerega GS, editor. Peritoneal surgery. New York: Springer-Verlag. 2000: 329-33.

10. DeWilde RL, Trew G. Postoperative abdominal adhesions and their prevention in gynaecological surgery. Expert consensus position.Gynecol Surg. 2007.

11. Duron JJ. Adhesive postoperative small bowel obstruction: incidence and risk factors of recurrence after surgical treatment: a multicenter prospective study. Ann Surg. 2006;244:750-7. 